\title{
Method to Calibrate Fission Chambers in Campbelling Mode
}

\section{ANIMMA International Conference}

\section{Benoit Geslot \\ Troy C. Unruh \\ Philippe Filliatre \\ Christian Jammes \\ Jacques Di Salvo \\ Stéphane Bréaud \\ Jean-François Villard}

The INL is a

U.S. Department of Energy

National Laboratory

operated by

Battelle Energy Alliance

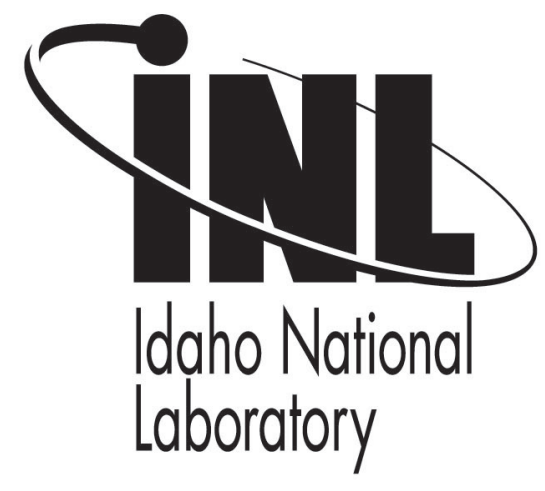

This is a preprint of a paper intended for publication in a journal or proceedings. Since changes may be made before publication, this preprint should not be cited or reproduced without permission of the author. This document was prepared as an account of work sponsored by an agency of the United States Government. Neither the United States Government nor any agency thereof, or any of their employees, makes any warranty, expressed or implied, or assumes any legal liability or responsibility for any third party's use, or the results of such use, of any information, apparatus, product or process disclosed in this report, or represents that its use by such third party would not infringe privately owned rights. The views expressed in this paper are not necessarily those of the United States Government or the sponsoring agency. 


\title{
Method to calibrate fission chambers in Campbelling mode
}

\author{
Benoit Geslot, Troy C. Unruh, Philippe Filliatre, Christian Jammes, Jacques Di Salvo, Stéphane Bréaud, Jean- \\ François Villard
}

\begin{abstract}
Fission chambers are neutron detectors which are widely used to instrument experimental reactors such as material testing reactors or zero power reactors. In the presence of a high level mixed gamma and neutron flux, fission chambers can be operated in Campbelling mode (also known as "fluctuation mode" or "mean square voltage mode") to provide reliable and precise neutron related measurements. Fission chamber calibration in Campbelling mode (in terms of neutron flux) is usually done empirically using a calibrated reference detector. A major drawback of this method is that calibration measurements have to be performed in a neutron environment very similar to the one in which the calibrated detector will be used afterwards. What we propose here is a different approach based on characterizing the fission chamber response in terms of fission rate. This way, the detector calibration coefficient is independent from the neutron spectrum and can be determined prior to the experiment. The fissile deposit response to the neutron spectrum can then be assessed independently by other means (experimental or numerical). In this paper, the response of CEA made miniature fission chambers in Campbelling mode is studied. We use a theoretical model of the signal to calculate the calibration coefficient. Input parameters of the model come from statistical distribution of individual pulses. Supporting measurements have been made in the CEA Cadarache zero power reactor MINERVE and results are compared to an empirical Campbelling mode calibration. The tested fission chamber calibration coefficient is about $21^{-26} \mathrm{~A}^{2} / \mathrm{Hz} /(\mathrm{c} / \mathrm{s})$. Both numerical and empirical methods give consistent results, however a deviation of about $15 \%$ was observed.
\end{abstract}

\section{INTRODUCTION}

$\mathrm{T}$ is generally accepted that fission chambers can be used

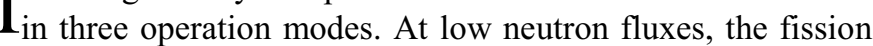
rate in the fissile deposit is low enough and fission products induced pulses are scarce(i.e. the average delay between two pulses is much larger than the pulse duration). They can be counted so as to obtain an event rate closely related to the detector fission rate: this is known as the "pulse mode". With the neutron flux increasing, pulses inside the chamber overlap and cannot be individually processed anymore. An indirect parameter related to the fission rate must be measured, namely the average current delivered by the detector in the "current

Manuscript received Mai 20, 2011.

B. Geslot is with the CEA, DEN, Cadarache, SPEx/LDCI, F-13108 S-Paullez-Durance, France (telephone: +33-4-42-25-2667, e-mail: benoit.geslot@cea.fr).

T. C. Unruh is with the Idaho National Laboratory, INL, P.O. Box 1625, MS 4112 Idaho Falls, ID 83415, USA (e-mail: Troy.Unruh@inl.gov).

P. Filliatre, C. Jammes, S. Bréaud and J-F. Villard are with the CEA, DEN, Cadarache, SPEx/LDCI, F-13108 Saint-Paul-lez-Durance, France.

J. Di Salvo is with the CEA, DEN, Cadarache, SPEx/LPE, F-13108 SaintPaul-lez-Durance, France. mode" or the variance of the current in the so-called "Campbelling mode". Indeed, if the process underlying the detection of neutrons is Poissonian, Campbell has demonstrated that both the current mean and variance are strictly proportional to the event rate [1].

The Cambelling mode is of great interest as it drastically diminishes the disturbance of gamma rays on fission chamber neutron signal [2][3].

Campbell theorems have been generalized to higher order statistics [4]. Recently, a new approach has been developed to model fission chamber signal [5]. It gives $n$-th order cumulants and spectra formula by using a model based on a random vector describing the current pulses (pulse shapes, deposited charge, etc.)

Our purpose here is to apply the same fission chamber modeling approach to the detector calibration in Campelling mode (i.e. for the second order). This work has been done in the framework of the research activities in fission chamber modeling conducted at CEA Cadarache [6].

The fission chamber calibration problem is described in section II. General formulas adapted for our measurement setup are given in section III. In section IV the experimental setup we used to validate our method is presented. We chose to irradiate a CEA-made miniature fission chamber in the zero power pool reactor MINERVE (Cadarache, France). Major experimental results are then presented and discussed in section $\mathrm{V}$.

\section{FISSION CHAMBER CALIBRATION}

The fission rate $R$ of a detector fissile deposit composed of $N$ fissile atoms can be related to the neutron flux $\Phi$ and to the overall fission cross section $\bar{\sigma}$ is expressed as:

$R / N=\bar{\sigma} \cdot \Phi$

By calculating the fission cross section, one can derive the on-line neutron flux. Inversely, by knowing the neutron flux, one can have access to an estimate of the fission cross section.

In the case of a detector with a thick fissile deposit, it is necessary to introduce an additional coefficient $k_{a}$ to take into account the signal loss due to the self-shielding (i.e. the neutron flux level depression mostly due to neutron absorption) and auto-absorption (accounted for by the stopping of fission products inside the deposit). Consequently, under the assumption that the "lost" fission products are not statistically different from the others, an "active fissile mass" composed of $N_{a}$ fissile atoms can be defined as: $N_{a}=k_{a} \times N$. So, the "active fission rate" can be defined as $R_{a}=N_{a} \cdot \bar{\sigma} \cdot \Phi$. 
$R_{a}$ is obviously the only quantity directly accessible to the measurement. The determination of the auto-absorption factor is not our purpose here, so in the following it will be assumed that the $k_{a}$ is equal to 1 (i.e. $R=R_{a}$ ).

Fig. 1 exhibits a general diagram describing fission chamber calibration shall now be presented. The relation between $R$ and $\Phi$ allows us to use a coefficient $K_{R}$ that depends solely on the neutron properties of the fissile deposit. A second coefficient $K_{d}$ is used to link the fission rate to the signal $S_{d}$ generated inside the detector. $K_{d}$ depends solely on the chamber response (gas mixture, pressure and geometry). Last, $K_{e}$ links the detector signal $S_{d}$ to the measurement $S_{e}$ and is related to the electronic system.

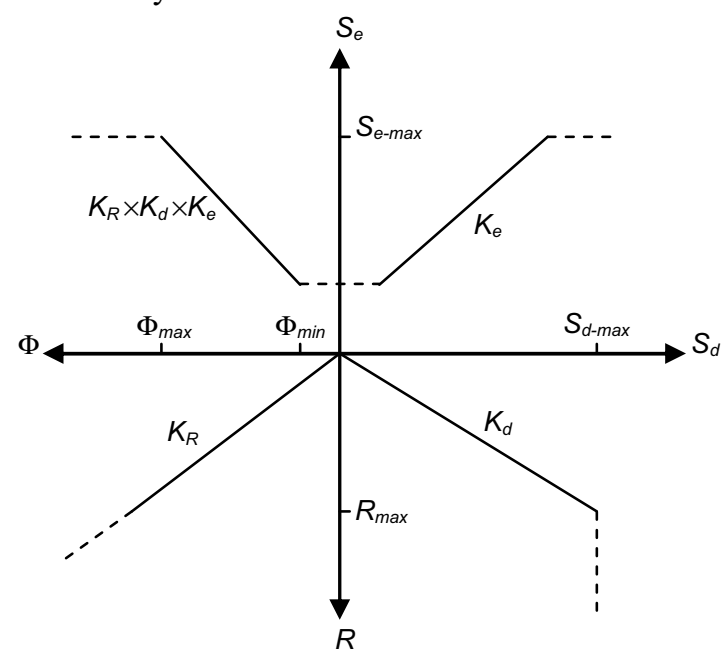

Fig. 1. A general and theoretical fission chamber calibration diagram. Calibration curves are to be read in log-log. Dotted lines represent lower and upper levels (i.e. beyond the operational range).

\section{CALIBRATION METHOD IN CAMPBELLING MODE}

In Campbelling mode, $S_{d}$ is the average amplitude of the power spectrum density of the output current. $S_{d}$ is expressed in $\mathrm{A}^{2} / \mathrm{Hz}$ and so $K_{d}$ is expressed in $\mathrm{A}^{2} / \mathrm{Hz} /(\mathrm{c} / \mathrm{s}) . K_{d}$ can be understood as the response of fission chamber to an average fission product emitted by the fissile deposit.

After the second Campbell theorem it is known that coefficient $K_{d}$ is related to the second order statistics of individual pulses occurring in the detector. We also know how to link the signal spectrum to the physical parameters of the detector [5].

As a result, one can show that the detector calibration factor is expressed as:

$K_{d}=\left(\bar{Q}^{2}+\sigma_{Q}^{2}\right) \cdot \frac{\Delta T}{F_{2}-F_{1}} \cdot \int_{F_{1}}^{F_{2}}|F T[\bar{h}]|^{2} d F$,

where $F T$ is the Fourier transform operator, $\bar{Q}$ (in C) and $\sigma_{Q}^{2}$ (in $\mathrm{C}^{2}$ ) are the first two moments of the collected charge statistics, $\bar{h}$ (expressed in $\mathrm{s}^{-1}$ ) is the average pulse at the output of the detector, $\Delta \mathrm{T}$ (in s) is the average pulse width and $\mathrm{F}_{2}-\mathrm{F}_{1}$ is the measurement frequency range (in $\mathrm{Hz}$ ).

This calibration method is based on acquiring individual pulses at the output of a fast broadband amplifier. Voltage signal has to be directly proportional to the current delivered by the detector. The signal to noise ratio has to be high enough so that the pulse discrimination does not greatly modify the statistical distributions of relevant parameters. Terms of (2) are calculated and reconstructed afterwards by straightforward numerical processing.

\section{EXPERIMENTAL SETUP}

MINERVE is a pool type ZPR operated by CEA Cadarache. The facility is dedicated to experimental programmes in support of the French nuclear industry and to improve neutron cross sections databases. Its maximum power is $80 \mathrm{~W}$. Two irradiation channels are available to test fission chambers in a nearly thermal neutron flux (about $10^{8} \mathrm{n} / \mathrm{cm}^{2} / \mathrm{s}$ at maximum power).

Our test detector is a miniature cylindrical CF4 type fission chamber manufactured at Cadarache Fission Chamber Workshop [7]. This detector (FC 2247) houses a $250 \mu \mathrm{g}$ deposit of enriched uranium (98.5\% U-235). An estimation of the deposit thickness is $0.6 \mu \mathrm{m}$. The detector outer diameter is $4 \mathrm{~mm}$ and the electrode gap is $0.5 \mathrm{~mm}$. The fill gas is pure argon at 12 bars.

Measurements in Campbelling mode were acquired using a new acquisition system called the Fast Neutron Detector System (FNDS). This system has been developed and qualified in the framework of the Instrumentation Joint Laboratory that regroups CEA and SCK $\cdot \mathrm{CEN}[8]$.

The FNDS acquisition system is sketched on Fig. 2. Its front end is a fast broadband current amplifier (gain of about $32500 \Omega$ ). The variance measurement is taken on a frequency range spanning from $20 \mathrm{kHz}$ to $300 \mathrm{kHz}$ (analog pass-band filter, $8^{\text {th }}$ order) and the acquisition frequency is $1 \mathrm{MHz}$. The electronics gain has been calibrated using synthesized signals: $K_{e}$ is about $10^{15} \Omega^{2} . \mathrm{Hz}$.

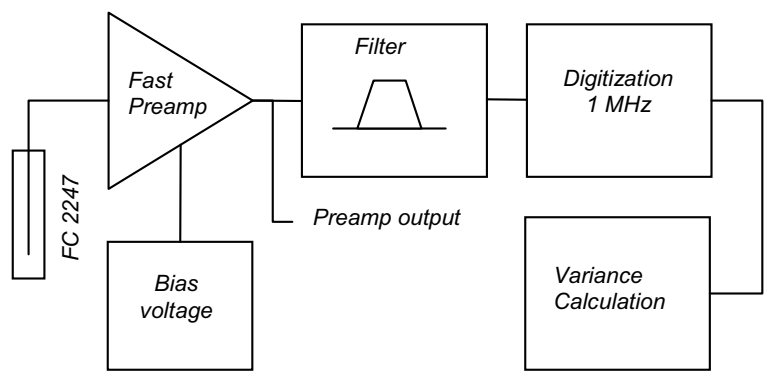

Fig. 2. Measurement setup in Campbelling mode using FNDS. The high immunity coaxial cable that links the detector to the preamplifier is about $25 \mathrm{~m}$ long.

\section{MAJOR RESULTS AND DISCUSSION}

\section{A. Calibration in pulse mode}

Our test detector signal was compared to the one of a calibrated fission chamber (FC 2232, same geometry, $25 \mu \mathrm{g}$ U-235). The measurement was done in the irradiation channel $\mathrm{n}^{\circ} 2$ at a power of $10 \mathrm{~W}$. Our calibrated detector gave us a fission rate of $R_{\text {/at }}=27.9 \mathrm{c} / \mathrm{s} / \mu \mathrm{g}$. The measurement is done using a standard Pulse Height Analysis channel (using Canberra spectroscopy electronic modules). 
FC 2247 PHA spectrum gives us a counting rate depending on the discrimination threshold (expressed as a fraction of a reference channel $\mathrm{C}$, see Fig. 3). We define the equivalent uranium mass as the mass that corresponds to a fission rate equal to the counting rate.

At low threshold, the noise level introduces a bias in the estimation of the counting rate (as we can see on Fig. 3, the counts increase below 0.1C). To circumvent that issue, we used an extrapolation of the equivalent mass down to a zero threshold. The obtained active uranium mass for FC 2247 is $219 \mu \mathrm{g}$. There is a $12 \%$ deviation between this mass and the total mass coated on the electrode (around $250 \mu \mathrm{g}$ ). This deviation can be explained by the auto-absorption effect [9].

By multiplying the active mass by the fission rate per atom, we can obtain the total event rate inside the detector. Based on this measurement, we will subsequently use an empirical factor to convert a counting rate obtained for a specific threshold into the total event rate. This factor is equal to 1.1 for a typical threshold of $0.3 \mathrm{C}$.

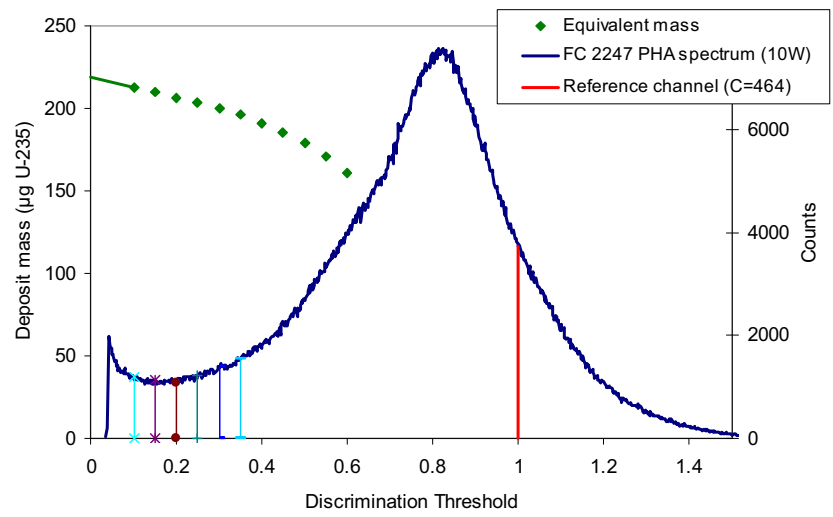

Fig. 3. FC 2247 PHA spectrum at $10 \mathrm{~W}$. The reference channel $\mathrm{C}$ is determined based on the spectrum maximum. An extrapolation of the spectrum below $0.1 \mathrm{C}$ allows us to estimate the active mass of the fissile deposit.

\section{B. Detector linearity with reactor power}

One of the main goals of the measurement campaign was to test the linearity of the detector by measuring the Campbelling mode signal at various reactor power levels. We tested 5 power levels from $1 \mathrm{~W}$ to $80 \mathrm{~W}$. The detector was installed in the irradiation channel $\mathrm{n}^{\circ} 2$ at core mid-plane and polarized at $300 \mathrm{~V}$.

At high power $(80 \mathrm{~W})$ a broadband spectrum of the signal at the FNDS preamplifier output was acquired on-line using an oscilloscope (LeCroy WaveRunner, $500 \mathrm{MHz}$ bandwidth and maximum sampling $5 \mathrm{GS} / \mathrm{s}$ ). FNDS signal spectrum was also calculated afterwards using samples of acquired signals. Both spectra are compared in Fig. 4.

The plot of the FNDS spectrum clearly shows the effect of the pass-band filter that eliminates the ionic signal part (below $20 \mathrm{kHz}$ ) is clearly observed. The frequency domain is nearly flat, which is in favor of the measurement steadiness.

FNDS variance measurements (in $\mathrm{V}^{2}$ ) were converted into power spectrum densities by using electronics calibration factor $K_{e}=10^{15} \Omega^{2}$.Hz. At each power level, a counting rate measurement was performed and converted into total event rates by using the pulse calibration factor.

Fig. 5 shows that the detector linearity is very good over nearly two decades. The first measurement point is close to the FNDS noise background (about $10^{-7} \mathrm{~V}^{2}$ ) and it was necessary to subtract the noise from the signal.

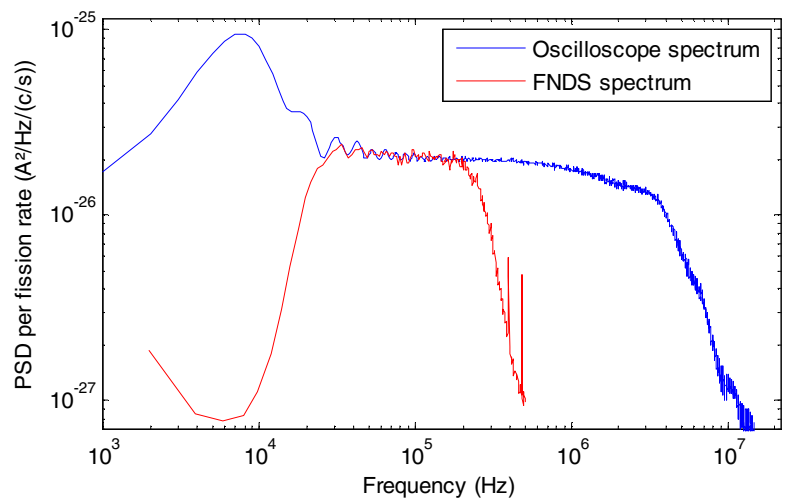

Fig. 4. FC 2247 spectra acquired with a broadband oscilloscope and with FNDS (frequency range $20 \mathrm{kHz}$ to $300 \mathrm{kHz}$ ).

The linear fit gives a empirical estimation of the calibration coefficient of $1.810^{-26} \mathrm{~A}^{2} / \mathrm{Hz} /(\mathrm{c} / \mathrm{s})$. The uncertainty of this result is difficult to assess since it depends mostly on the pulse calibration factor. We estimate that it is around $10 \%(68 \%$ confidence interval).

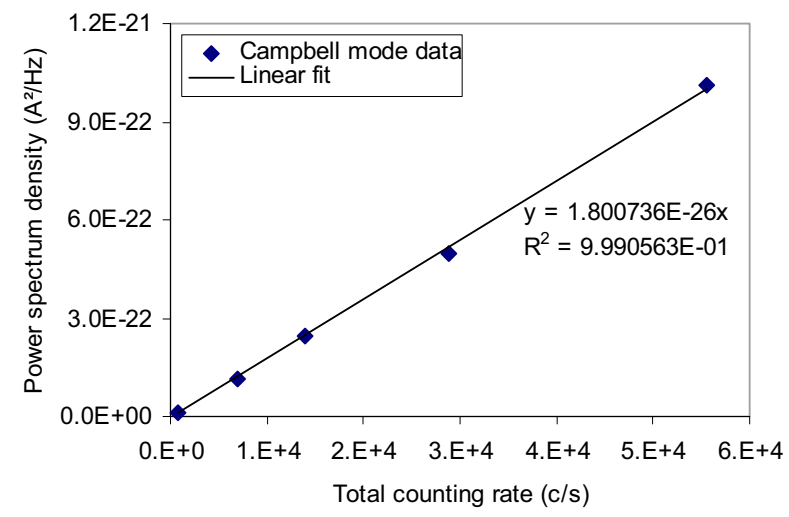

Fig. 5. Campbelling mode signal acquired by FNDS versus the total event rate in the FC 2247. The linear fit slope gives us an estimation of the Campbelling mode calibration factor $K_{d}$.

\section{Pulses measurements}

Detector signals were acquired at FNDS preamplifier output in order to access statistical properties of delivered pulses (collected charge, pulse width, pulse shapes). Our objective here is to estimate every parameters needed to apply (2).

Individual pulses were acquired at low power $(10 \mathrm{~W})$ using the oscilloscope. The event rate in the detector is lower than $10^{4}$ pulses per second. Fig. 6 shows the detector average pulse as well as the pulse shape standard deviation (upper plot). The charge spectrum was calculated offline (lower plot). It is found to be is very consistent with the one obtained by a standard PHA measurement.

Main calculated parameters of the average pulse are summarized in Table I. As it can be seen, the average charge 
coming from the electronic component of the pulse is not equal to the charge collected from the ionic part. That comes from the fact that the charges are not uniformly created in the electrode gap. On the contrary, charges are mostly created close to the fissile deposit, so the electrons' path in the gas is on average smaller than the ions path.

The sum of the electronic and ionic charges gives us the total charge $\bar{Q}$ deposited by an average fission product in the detector. This quantity is often expressed in terms of number of electrons. In our case, we have on average $1.410^{6}$ electrons by fission product. Charge standard deviation $\sigma_{Q}$ was found to be $33 \%$ of the average charge.

\section{TABLE I. FC 2247 PULSE PARAMETERS}

\begin{tabular}{lll} 
Parameter & Electronic component & Ionic component \\
\hline Width & $150 \mathrm{~ns}$ & $40 \mu \mathrm{s}$ \\
Amplitude & $1.4 \mu \mathrm{A}$ & $7.2 \mathrm{nA}$ \\
Charge & $91 \mathrm{fC}$ & $132 \mathrm{fC}$ \\
\hline
\end{tabular}

Using the parameters in Table I and by numerically computing the Fourier transform of the pulse shape, we were able to calculate the calibration coefficient solely based on pulse measurements.
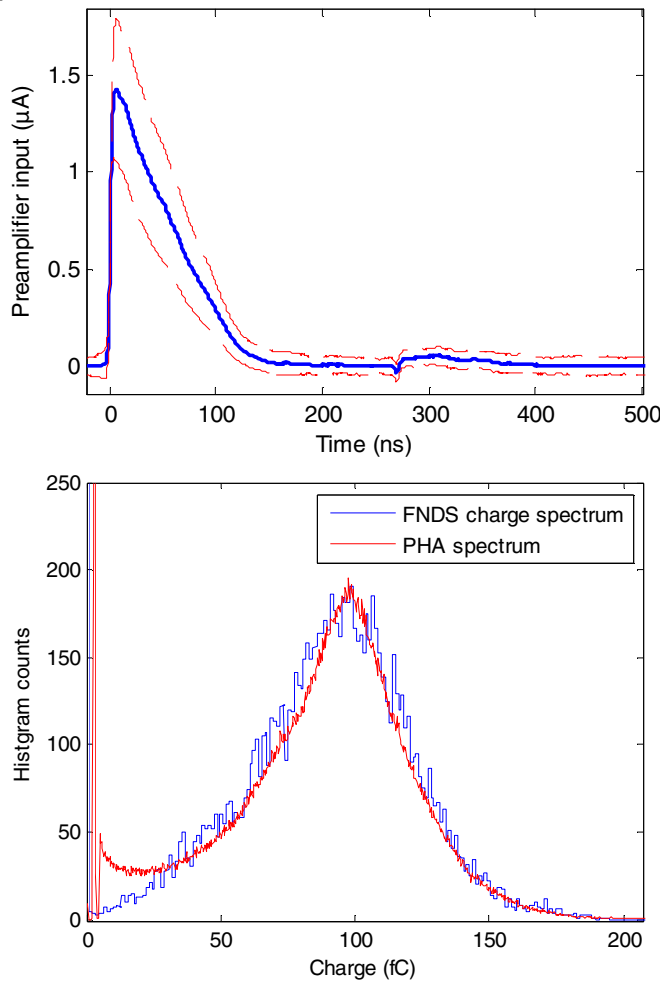

Fig. 6. Average pulse acquired at FNDS preamplifier output (upper plot). Charge spectrum (lower plot) based on individual pulses analysis (blue) and measured using a standard PHA channel (red).

Our calculation gave us a $K_{d}$ equal to $2.0510^{-26} \mathrm{~A}^{2} / \mathrm{Hz} /(\mathrm{c} / \mathrm{s})$. That value is consistent with the empirical value of $1.810^{-26}$ $\mathrm{A}^{2} / \mathrm{Hz} /(\mathrm{c} / \mathrm{s})$. The deviation of $14 \%$ between the two values can most likely be attributed to the imperfections of our estimation of the detector total counting rate. Another source of uncertainty is the poor signal to noise ratio (around 10) that we had when acquiring pulses at FNDS preamplifier output.

\section{CONCLUSION}

In this paper a method is detailed to calibrate fission chambers in Campbelling mode. It is based on characterizing the detector pulses and calculating the detector response using a detailed expression of Campbell's second theorem.

Results acquired at the MINERVE reactor using a CEA made miniature fission chamber with $250 \mu \mathrm{g}$ uranium deposit demonstrated the feasibility of the method. The obtained calibration coefficient is $2.0510^{-26} \mathrm{~A}^{2} / \mathrm{Hz}$. A $14 \%$ deviation between this value and the reference one based on a calibration of the detector in pulse mode was observed.

In a subsequent work, we plan to further test the robustness of the method and apply it to other miniature fission chambers with various technological parameters (gas mixture, pressure and fissile deposit).

\section{REFERENCES}

[1] N. R. Campbell and V. J. Francis, "A theory of valve and circuit noise", Journal of the Institution of Electrical Engineers, vol. 93, n. 3, 1946.

[2] L. Vermeeren, M. Weber, L. Oriol, S. Breaud, P. Filliatre, B. Geslot, C. Jammes, S. Normand and B. Lescop, "Experimental Verification of the Fission Chamber Gamma Signal Suppression by the Campbelling Mode", IEEE Transactions on Nuclear Science, vol. 58, 2011, p. 362369.

[3] D. Fourmentel, J-F. Villard et al. "Combined analysis of neutron and photon flux measurements for the Jules Horowitz Reactor core mapping", $2^{\text {nd }}$ Internation Conference on Advancements in Nuclear Instrumentation Measurement Methods and their Applications (ANIMMA), 2011,

[4] I. Lux and A. Baranyai, "Higher order campbell techniques for neutron flux measurement: I. Theory", Nucl. Instr. Meth., vol. 202, n'. 3, p. 469475, nov. 1982.

[5] E. W. Pontes et A. Ferreira, "Using cumulants and spectra to model nuclear radiation detectors", IEEE transactions on nuclear science, vol. 53, no. 3, p. 1292-1298, 2006.

[6] C. Jammes, P. Filliatre, B. Geslot, L. Oriol, F. Berhouet, J.-F. Villard, et L. Vermeeren, "Research Activities in Fission Chamber Modeling in Support of the Nuclear Energy Industry", IEEE Transactions on Nuclear Science, 2010.

[7] B. Geslot, F. Berhouet, L. Oriol, S. Bréaud, C. Jammes, P. Filliatre, et J.F. Villard, "Development and manufacturing of special fission chambers for in-core measurement requirements in nuclear reactors", Advancements in Nuclear Instrumentation Measurement Methods and their Applications (ANIMMA), 2009.

[8] B. Geslot, L. Vermeeren, P. Filliatre, A.L. Lopez, L. Barbot, C. Jammes, S. Bréaud, L. Oriol, et J.-F. Villard, "New measurement system for on line in core high-energy neutron flux monitoring in materials testing reactor conditions", Rev. Sci. Instr., vol. 82, 2011, p. 033504.

[9] C. Jammes, P. Loiseau, P. Filliatre, B. Geslot "Uranium coating impact on fission chamber signal". To be published 